\title{
The reality of the wavefunction: old arguments and new*
}

\author{
Harvey R. Brown ${ }^{\dagger}$
}

April 9, 2017

\begin{abstract}
Faculty of Philosophy, University of Oxford, Radcliffe Humanities, Woodstock Road, Oxford OX2 6GG, UK.
\end{abstract}

We show that not only individual atoms but matter in bulk would [in the absence of the Pauli exclusion principle] collapse into a condensed highdensity phase. The assembly of any two macroscopic objects would release energy comparable to that of an atomic bomb. Freeman Dyson ${ }^{1}$

Thus our daily experience that 2 liters of gasoline contain only twice as much energy as 1 litre is a pathological property of small clumps of matter containing fermions. ... For fermi-matter only objects somewhat heavier than our sun are doomed to gravitational collapse but if mountains were made of bose-matter they would crush under their own weight. Walter Thirring ${ }^{2}$

\footnotetext{
Abstract

The recent philosophy of Quantum Bayesianism, or QBism, represents an attempt to solve the traditional puzzles in the foundations of quantum theory by denying the objective reality of the quantum state. Einstein had hoped to remove the spectre of nonlocality in the theory by also assigning an epistemic status to the quantum state, but his version of this doctrine was recently proved to be inconsistent with

*Forthcoming in Ontology Studies - Outstanding Papers from the San Sebastian International Congresses of Ontology.

${ }^{\dagger}$ harvey.brown@philosophy.ox.ac.uk

${ }^{1}$ Dyson (1967).

${ }^{2}$ Thirring (1986), p. 345.
} 
the predictions of quantum mechanics. In this essay, I present plausibility arguments, old and new, for the reality of the quantum state, and expose what I think are weaknesses in QBism as a philosophy of science.

\section{Contents}

1 Non-realist interpretations of the wavefunction 2

2 Wider concerns $\quad 5$

3 Remarks on QBism $\quad 14$

4 Acknowledgments $\quad 26$

$\begin{array}{llr}5 & \text { References } & 26\end{array}$

\section{Non-realist interpretations of the wavefunction}

Whatever the quantum mechanical wavefunction is, it is not fundamental. The wavefunction $\psi$ and its unitary dynamics are emergent elements within relativistic quantum field theory (RQFT), associated with the nonrelativistic, low energy regime. ${ }^{3}$ But this state of affairs is no impediment in principle to the reality of $\psi$, or more generally the statistical (density) operator. ${ }^{4}$

Arguments for the non-reality of the wavefunction take various forms. Some prominent advocates of the de Broglie-Bohm pilot wave theory argue that the quantum state can be thought of as part of the laws of nature, with a status akin to that of the Hamiltonian. Adoption of such a nomic view is critical in rebutting the criticism that the theory is essentially Everettian quantum theory in denial. I will not repeat here arguments which Wallace and collaborators have advanced which question the Hamiltonian analogy. ${ }^{5}$ The considerations in section 2 below complement these arguments.

\footnotetext{
${ }^{3}$ See, e.g., Wallace and Timpson (2010) and Myrvold (2014).

${ }^{4}$ I will bypass here the debate between realists about the quantum state regarding whether the state should be defined on configuration space (see e.g. Ney (2015)) or (nonseparably) on space (see Wallace and Timpson op.cit.).

${ }^{5}$ See Wallace and Timpson op.cit. and Brown and Wallace (2005). The strongest arguments for the nomic reading of the wavefunction in my opinion are found in Callender (2017), which build on the case made by Dürr et al. (1997), and address the criticism in Brown and Wallace ibid. In this connection see also Maudlin (2010).
} 
A prominent advocate of the alternative epistemic view of the quantum state is Christopher Fuchs.

... the quantum state represents a collection of subjective degrees of belief about something to do with that system (even if only in connection with our experimental kicks to it) ...

Our foremost task should be to go to each and every axiom of quantum theory and give it an information theoretic justification if we can ...

Quantum states are states of information, knowledge, belief, pragmatic gambling commitments, not states of nature. ${ }^{6}$

Such a view has prime facie a lot going for it. If it is right, then it would seem that the notorious collapse of the wavefunction in the act of measurement is innocuous: it corresponds to nothing other than Bayesian updating. ${ }^{7}$ As a consequence, the threat of instantaneous action-at-a-distance in the 1935 Einstein-Podolsky-Rosen (EPR) scenario involving entangled systems is also removed. ${ }^{8}$

If only things were so simple!

Let us start with the well-known, and surely most obvious, articulation of the $\psi$-epistemic view which I shall call the Einstein version. To borrow Fuch's words, Einstein suggested from at least as early as 1929 that "the quantum state represents a collection of subjective degrees of belief about something to do with that system". The "something" in Einstein's understanding was the hidden, ontological state of the system. Einstein, unlike Fuchs, was proposing a deterministic hidden variable theory of a certain kind, precisely in the hope that it would remove not only what he saw as the spectre of non-locality in othodox quantum mechanics (QM). ${ }^{9}$ but also the "paradox" involved in obtaining definite outcomes in generic measurement procedures ${ }^{10}$ - essentially what is known today as the measurement problem.

The prospects of the Einstein version of the $\psi$-epistemic view look very bleak. Starting with the work of Pusey, Barrett and Rudolph (PBR) in 2012, a series of no-go proofs have appeared in the literature, which show, on the basis of plausible auxiliary assumptions, that the Einstein version is

\footnotetext{
${ }^{6}$ Fuchs (2002a).

${ }^{7}$ See Fuchs et al. (2014) and Leifer (2014), p. 68.

${ }^{8}$ See Fuchs et al. (2014) and Timpson (2008).

${ }^{9}$ See Harrigan and Spekkens (2010).

${ }^{10}$ This is particularly clear in Einstein (1970), pp. 670 and 683.
} 
inconsistent with the predictions of quantum mechanics. ${ }^{11}$ But even before these recent dramatic results were obtained, there were grounds for doubting the success of the Einstein version as a solution of both the measurement and nonlocality problems. It has long been known that the process of measurement must, in general, disturb the hidden state (if any) of the system in question, whatever view is taken on the status of the wavefunction in the theory. ${ }^{12}$ Whether this disturbance is compatible with the inter-measurement dynamics would depend on the details of the theory and cannot be guaranteed a priori. $^{13}$ In relation to the EPR challenge, I refer of course to the many non-locality theorems inspired by the 1964 work of J. S. Bell, and to a great deal of subsequent experimentation, which together show that any deterministic hidden variable theory must incorporate action-at-a-distance if it is consistent with the proven predictions of QM. ${ }^{14}$

The so-called Copenhagen interpretation is widely understood to deny a realist status to the quantum state, which is nonetheless taken to be a complete description of the system. The state is a mathematical tool within the quantum algorithm, allowing for probabilistic predictions to be made concerning the outcome of measurements involving macroscopic instruments which themselves can and must be described "classically". I have no intention of rehearsing all the well-known challenges facing this interpretation, in so far as it can be regarded as a single thing. But it will be useful to remind ourselves of the stinging criticism John Bell raised against it in 1990:

To restrict quantum mechanics to be exclusively about piddling laboratory operations is to betray the great enterprise. A serious formulation will not exclude the big world outside the laboratory. ${ }^{15}$

Need this exhortation have as its target all versions of the $\psi$-epistemic view? Not according to Fuchs and collaborators: their relatively recent philosophy of Quantum Bayesianism, or QBism, is, they claim, an exception. I use the word philosophy advisedly. In its attempt to resolve the puzzles of quantum mechanics, QBism makes the jaw-dropping claim to "liberate us from the grip of an ancient Greek maneuver that worked for

\footnotetext{
${ }^{11}$ A detailed review of these recent results is found in Leifer (2014).

${ }^{12}$ For details see Squires et al. (1994), p. 429.

${ }^{13}$ The de Broglie-Bohm theory suffers from no such incompatibility, but it is not a $\psi$-epistemic theory of the Einstein version.

${ }^{14}$ For a recent comprehensive collection of essays on this matter, see Bell and Gao (2016).

${ }^{15}$ Bell (1990).
} 
over two millennia", ${ }^{16}$ to overturn the allegedly dominant natural philosophy in which science has an "object" but not a "subject". These heady matters deserve special attention, and I will return to QBism in section 3 of this essay. For the moment I note that since QBism denies that the "something" the quantum state refers to probabilistically is itself an element of observer-independent reality, the theory not only claims to solve both the measurement and nonlocality problems ${ }^{17}$, it also survives the recent PBRtype no-go results. ${ }^{18}$ But at what cost? ${ }^{19}$

\section{Wider concerns}

When John Bell in 1966, and Simon Kochen and Ernest Specker in 1967, independently proved that non-contextual hidden variable theories are inconsistent, there was little to indicate that such a result was likely within the prior literature on the foundations of quantum mechanics. ${ }^{20}$ The post-2012 no-go results concerning Einstein's $\psi$-epistemic position, impressive as they are, surely are not as surprising. Powerful plausibility arguments have long been available, some since the birth of QM, to the effect that the quantum state is something real. They almost all have to do, in one way or another, with quantum phase, with the fact that the wavefunction, in its relation to probability, is strictly a (generally complex) probability amplitude: it has more structure than a probability distribution does.

\section{$2.1 \quad$ Interference}

Arguably the oldest and most striking of these plausibility arguments is based on interference effects. Whether it be the old chestnut, the two-slit experiment (in both its spatial, and less well-known temporal variants ${ }^{21}$ )

\footnotetext{
${ }^{16}$ Fuchs et al. (2014).

${ }^{17}$ See Fuchs et al. (2014) and Timpson (2007).

${ }^{18}$ See Pusey et al. (2012) and Leifer (2014), section 14.4. For details of advocates of such $\psi$-epistemic views other than the authors of QBism, see ibid p. 72, and Healey (2016), which also contains a useful review of QBism and its history. Healey's own "pragmatist" approach of the wavefunction (for details see ibid) has much in common with QBism but important differences as well.

${ }^{19}$ The following section of this paper is an attempt to make the case for the realist interpretation of the wavefunction; a more elaborate discussion is found in Gao (2017).

${ }^{20}$ David Bohm's 1952 hidden variable theory had already shown that von Neumann's 1932 no-go result was inconclusive.

${ }^{21}$ For a striking experimental version of the latter involving atomic interferometry, see Szriftgiser et al. (1996). For an experimental proposal involving neutrons, with references to earlier optical variants, see Brown et al. (1992).
} 
or the Mach-Zender interferometer for photons, or neutron or atomic interferometers, such displays of of single-system interference effects cry out for a realist interpretation of the wavefunction. Of course the case is not completely water-tight, as Leifer has recently stressed.

Interference phenomena also occur in [certain toy models] simply because they reproduce fragments of quantum theory exactly and those fragments contain coherent superpositions. It is arguable whether the mechanisms explaining interference in all these models are plausible, but the main point is that the direct inference from interference to the reality of the wavefunction is blocked by them. If there is an argument from interference to be made then it will need to employ further assumptions. ${ }^{22}$

Although not what Leifer had in mind, a particularly intriguing recent example is a fluid mechanical ("walking droplet") model of diffraction, tunneling, quantisation and other quantum-like effects. ${ }^{23}$

Neither this nor the toy models Leifer is referring to capture all of the quantum predictions, and a striking omission is entanglement and its manifold manifestations. (Of particular interest for our purposes is the antisymmetric nature of the many-electron wavefunction, of crucial importance in accounting for the stability of bulk matter; see below.) So perhaps an analogy will help in addressing Leifer's skepticism. Consider the explanation of the gravitational redshift phenomenon in general relativity. Although in the actual experimental confirmations of this phenomenon tidal effects are negligible, the explanation refers to a metric field with curvature, a solution of Einstein's field equations. Would it not be odd to cast doubt on this explanation just because the experimental redshift phenomenon can also be explained in flat spacetime ${ }^{24}$ It is common scientific practice that an explanation for a given physical phenomenon is provisionally accepted when the theory behind it is uniquely capable of accounting for a wide gamut of diverse phenomena, even when in relation to the given phenomenon it may not provide the only explanation. ${ }^{25}$

\footnotetext{
${ }^{22}$ Leifer op. cit., p. 79 .

${ }^{23}$ See Bush (2015) and further references therein.

${ }^{24}$ That (first order) redshift is consistent with flat Minkowski spacetime has long been known, but it is not always acknowledged; for details see Brown and Read (2016).

${ }^{25}$ Attempts to describe all known gravitational effects in a theory based on flat spacetime generally turn out to be awkward reformulations of general relativity, and I suspect that any future "toy" model that accounted for more than a fragment of quantum theory would likewise be an awkward reformulation of that theory.
} 
I would particularly like to mention the case of partial absorption experiments in single neutron interferometry which were performed by Helmut Rauch and his collaborators in Vienna in the 1980s. In one experiment, a rotating toothed wheel, or "chopper", constructed out of fully absorbing material (cadmium), is placed in one of the two beams inside the interferometer; it deterministically absorbs a certain percentage of the successive neutrons "in" that beam, and in doing so changes (weakens) the interference pattern recorded in the beams of (unabsorbed) neutrons emerging from the interferometer. In the other experiment, a static piece of gold foil replaces the chopper; the nuclei in the new absorber will likewise absorb a certain percentage of the neutrons inside the interferometer, but this process is intrinsically probabilistic. The experiments corroborate the prediction in quantum mechanics that even when the absorption coefficients are the same in both cases, and therefore so is the Shannon information concerning which beam the neutron is "in", the interference effects are different - there is a greater degree of interference in the case of the gold foil. (A third hypothetical example involves a slit in partially absorbing material; again the Shannon information can be arranged to be the same as in the previous examples, but the loss of interference will be intermediate, depending on the penetration of the neutron wavepacket in the slit material. ${ }^{26}$ )

Finally, certain quantum interference experiments involving electrons and neutrons provide strong, if not conclusive, grounds for supposing that the properties of mass (inertial and gravitational), charge and magnetic moment adhere to the wavefunction itself $^{27}$ - if this is not already seen to follow from the simple fact that such properties appear in its equation of motion. It would seem to follow that in both the nomic version of de Broglie-Bohm theory and QBism, such properties have no describable observer-independent physical entities in which to reside.

\subsection{Phase matters}

In an important paper of 1962, Merzbacher investigated the conditions in quantum mechanics required for the quantization of angular momentum for a spinless particle. ${ }^{28}$ In particular, he was concerned to show that the singlevaluedness of the wavefunction is one of the conditions, as it is in the derivation of the original Aharonov-Bohm effect. Merzbacher demonstrated that single-valuedness itself is motivated when the background space (whether 2

\footnotetext{
${ }^{26}$ For further details on all these cases, see Kaloyerou and Brown (1999).

${ }^{27}$ See Brown et al. (1995)

${ }^{28}$ Merzbacher (1962).
} 
or 3-dimensional) is simply connected. In the case of a system of identical particles, where the wavefunction is defined on configuration space, or rather the reduced space obtained by identifying the configuration points related by particle permutations, the topology of the reduced space is again important, as Leinaas and Myrheim demonstrated in $1977 .{ }^{29}$ If points corresponding to two or more particles coinciding spatially are excised from the space, so that it becomes non-simply connected, the wavefunction is no longer singlevalued. The upshot is that if the physical space in which the particles live has three or more dimensions, then the wavefunction can be shown to be either symmetric or antisymmetric under permutations of particle labels. This constraint is widely regarded as a postulate in standard quantum mechanics, but here it is derived on topological grounds. Even more remarkably, if the physical space is two dimensional, intermediate phase factors between 1 and -1 are possible under permutations, and this leads to the possibility of 'fractional' or 'braid' statistics ranging between Bose-Einstein and FermiDirac. This is not a mere theoretical oddity. It is apparently displayed in two-dimensional electron gases in a transversal external magnetic field exhibiting the fractional quantum Hall effect. ${ }^{30}$ Certain systems exhibiting the fractional quantum Hall effect are being investigated with a view to application in quantum computation.

I do not claim that such considerations are outright inconsistent with the $\psi$-epistemic position. But it is again unclear to me how the topology of physical space in the case of single particles, and the topology of the reduced configuration space as well as the dimensionality of physical space in the case of the many (identical) particles system, can be understood to play such important roles in determining critical properties of the wavefunction within this interpretation.

\subsection{The stability of matter}

In his systematic 2014 review of no-go theorems for $\psi$-epistemic theories, Leifer referred to what he called the neo-Copenhagen views which, like QBism, reject the notion that the wavefunction is a probability distribution over ontic states. He wrote in this connection:

For my part, I think that if one denies the existence of an observer-independent reality then it becomes very difficult to

\footnotetext{
${ }^{29}$ Leinaas and Myrheim (1977).

${ }^{30}$ See, e.g., Prange and Girvin (1990). It is notable that space reflections and time reversal are not symmetries of such electron gases. See Frohlich (2009), p. 56.
} 
maintain a clear notion of explanation at all. Closing explanatory gaps by denying the need for any explanation at all does not seem that appealing to me. ${ }^{31}$

These remarks arguably do not do justice to the role of the external world in QBism (see section 3(v) below), but the point is well taken. In his earlier detailed 2008 study of QBism, Christopher Timpson had also raised the issue of an "explanatory deficit" in the theory. ${ }^{32}$ He questioned whether, for example, the standard explanation in quantum theory of the thermal and electrical conductivity properties of solid matter, can be incorporated into QBism. Timpson's core point was that the QBist can explain why someone would believe that, for example, matter conducts but cannot explain why matter does conduct. He also mentioned in this context the explanation of the stability of matter, but did not provide details. I intend in this section to provide some of these details, in the spirit of Timpson's critique. ${ }^{33}$

(i) It is a remarkable fact that a satisfactory quantum mechanical explanation of the stability of bulk matter emerged only in 1967. But let us consider the single-electron atom/ion first. Here, the proof of stability is older, but the full story is still often omitted from textbooks. Quantum mechanics explains the stability of discrete spectral lines (modulo a satisfactory solution to the "measurement problem"!), which were of course mysterious from a classical perspective. But it is of greater significance that the theory accounts for the fact that the energy of the electron is bounded from below. The key challenge is the nature of the $1 / r$ Coulomb electrostatic potential, as Jeans had noted in 1915. ${ }^{34}$ Bound electrons have negative potential energy. What is to prevent the electron from getting arbitrarily close to the nucleus, so that its potential energy approaches negative infinity, while its kinetic energy remained arbitrarily small? Were this to happen, in the words of Elliott Lieb,

...the hydrogen atom would be physically unstable; in a gas of many atoms another particle or atom could collide with our atom and absorb energy from it. After many such collisions our electron could find itself in a tiny orbit around the nucleus and our atom would no longer be recognizable as an object whose

\footnotetext{
${ }^{31}$ See Leifer (2014), p. 139.

${ }^{32}$ Timpson (2008).

${ }^{33}$ I will restrict myself to non-relativistic quantum mechanics; the relativistic version of the story of stability can be found in Lieb and Seiringer (2010).

${ }^{34}$ See Lieb (1990), p. 7.
} 
radius is supposed to be $10^{8} \mathrm{~cm}$. Each atom would be an infinite source of energy which could be transmitted to other atoms or to radiation of electromagnetic waves. ${ }^{35}$

One can solve the time-independent Schrödinger equation for the hydrogen atom to show that the ground state energy is finite, but this procedure is unfeasible for large atoms and a simpler, generalisable one is desirable. To this end, a variant of the Heisenberg uncertainty relation is often employed. Consider the kinetic energy $T=p^{2} / 2 m=-\hbar^{2} \Delta / 2 m$ and its expectation value for any particle of mass $m$ and wavefunction $\psi$ :

$$
\langle T\rangle_{\psi}=\frac{\hbar^{2}}{2 m}(\psi,-\Delta \psi)=\frac{\hbar^{2}}{2 m} \int_{\mathbb{R}^{3}}|\nabla \psi(\mathbf{x})|^{2} \mathrm{~d} \mathbf{x} .
$$

The Heisenberg uncertainty relation is, then, for any $\psi$ of unit norm,

$$
\langle T\rangle_{\psi}\left\langle x^{2}\right\rangle_{\psi} \geq \frac{9 \hbar^{2}}{8 m}
$$

where

$$
\left\langle x^{2}\right\rangle_{\psi}=\int_{\mathbb{R}^{3}} x^{2}|\psi(\mathbf{x})|^{2} \mathrm{~d} \mathbf{x} .
$$

The inequality (2) means in this case that increasing localisation of $\psi$ around the origin (the nucleus) is associated with a correspondingly large value of the kinetic energy, so stability of the atom is secured. But the argument fails if, for example, $\psi$ has two "bumps", one localised around the nucleus and containing most of the mass, and the other localised at, say, the moon. In this case, $\left\langle x^{2}\right\rangle_{\psi}$ is large, so $\langle T\rangle_{\psi}$ can be small, while the average potential energy decreases without bound.

Fortunately help is at hand. In 1938 Sobolev proved the following inequality: ${ }^{36}$

$$
\langle T\rangle_{\psi} \geq \frac{3 \hbar^{2}}{2 m}\left(\frac{\pi}{2}\right)^{\frac{4}{3}}\left\{\int_{\mathbb{R}^{3}} \rho_{\psi}(\mathbf{x})^{3} \mathrm{~d} \mathbf{x}\right\}^{\frac{1}{3}},
$$

where $\rho_{\psi}(\mathbf{x})=|\psi(\mathbf{x})|^{2}$. It can be shown that when $\psi$ is of unit norm, it follows from the Sobolev inequality that the mean value of the ground state energy of the Hydrogen atom is bounded from below. ${ }^{37}$

\footnotetext{
${ }^{35}$ Lieb (1990).

${ }^{36}$ Sobolev (1938). We are concerned here with the three-dimensional version of the original inequality. For further details see Seiringer (1990) section 1.3.

${ }^{37}$ See Lieb (1976), section 1, Lieb (1990) Part III, and Seiringer (1990), section 1.4. It should not be concluded however that a proof of this kind of the stability of the hydrogen atom was only possible in 1938, with the appearance of the Sobolev inequality. A weaker, but less useful inequality due to Hardy (1920) suffices; see, e.g., Seiringer (1990) and particularly Frank (2011).
} 
Now a special case of the Hölder inequality ${ }^{38}$ states

$$
\int_{\mathbb{R}^{3}} \rho_{\psi}(\mathbf{x})^{\frac{5}{3}} \mathrm{~d} \mathbf{x} \leq\left\{\int_{\mathbb{R}^{3}} \rho_{\psi}(\mathbf{x})^{3} \mathrm{~d} \mathbf{x}\right\}^{\frac{1}{3}}\left\{\int_{\mathbb{R}^{3}} \rho_{\psi}(\mathbf{x}) \mathrm{d} \mathbf{x}\right\}^{\frac{2}{3}},
$$

so assuming as before that $\psi$ has unit norm (so the second term on the RHS of (5) is unity), applying (5) to the Sobolev inequality yields

$$
\langle T\rangle_{\psi} \geq \frac{3 \hbar^{2}}{2 m}\left(\frac{\pi}{2}\right)^{\frac{4}{3}} \int_{\mathbb{R}^{3}} \rho_{\psi}(\mathbf{x})^{\frac{5}{3}} \mathrm{~d} \mathbf{x} .
$$

Elliott Lieb expresses the content of this inequality "poetically" as follows:

An electron is like a rubber ball, or a fluid, with an energy density proportional to $\rho_{\psi}^{5 / 3}$. It costs energy to squeeze it and this accounts for the stability of atoms. ${ }^{39}$

This is also the fundamental reason why dynamical collapse models of QM involve non-conservation of energy and momentum. For the QBist, however,

The notorious "collapse of the wave-function" is nothing but the updating of an agent's state assignment on the basis of her experience. $^{40}$

It is not clear to me how easy it is to reconcile this claim with the fact that in the case of a localisation measurement, collapse is accompanied by a change in the expected energy of the system.

(ii) An even more profound analogue of the inequality (6) holds in the case of bulk matter, containing many electrons, protons and neutrons. Not surprisingly the details in this case are far more complicated; I shall do no more than sketch the main results.

Consider the ground state energy $E_{0}$ of a system comprised of $N$ electrons and $M$ nuclei, defined by

$$
E_{0}=\inf \{(\Psi, H \Psi):\|\Psi\|=1, \Psi \in \mathcal{H}\},
$$

where $H$ is the Hamiltonian associated with the system and $\mathcal{H}$ is the Hilbert space of possible states $\Psi$. The Hamiltonian contains Coulombic terms

\footnotetext{
${ }^{38}$ For further details see Lieb (1976), p. 555, or Seiringer (1990), p. 9.

${ }^{39}$ Lieb (1990). Note that none of the considerations here require that the wavefunction be complex.

${ }^{40}$ Fuchs et al. (2014).
} 
describing the attraction of the nuclei and electrons, the repulsion between the electrons and the repulsion between the nuclei. Because the proton mass is three orders of magnitude greater than that of the electron, the nuclei can be treated as classical objects at fixed locations, and it is the many-electron wavefunction that is the object of study:

$$
\Psi=\Psi\left(\mathbf{x}_{1}, \sigma_{1} ; \ldots ; \mathbf{x}_{N}, \sigma_{N}\right),
$$

where the space variables $\mathbf{x}_{i}$ range over $\mathbb{R}^{3}$, and the spin variables can take $q$ values. (For electrons the $\sigma_{i}$ take values in $\{-1 / 2,1 / 2\}$, so $q=2$.)

The first issue associated with the stability of bulk matter is, again, how to avoid of the possibility of implosion. As with the case of the individual atom, the ground state energy $E_{0}$ must be bounded from below: $E_{0}>$ $-\infty$. This is called stability of the first kind. But we also require that $E_{0}$ satisfy another inequality: $E_{0} \geq-C(N+M)$, where $C$ is non-negative and independent of $N$ and $K$; it depends on the maximum positive charge on the nuclei. This is called stability of the second kind. The reason for this requirement needs to be spelt out.

When we mix two equal quantities of (say) water together, we expect to the quantity of water to double, without the release of any significant amount of energy. But the terms in the Coulomb interaction quadruple, and the electrostatic energy grows with the square of the number $N+M$, not linearly. ${ }^{41}$ Now the total ground state energy is $2 E_{0}(N+M)$ before mixing. After mixing, the ground state energy becomes $E_{0}(2(N+M))$ so the energy released will be $\Delta E_{0}=2 E_{0}(N+M)-E_{0}(2 N+M)$. So suppose that the energy content of matter made is proportional to (minus) the square of the number of particles $N+M$. Then on mixing the water, an energy proportional to $2(N+M)^{2}$ would be released, where $N+M$ is of the order $10^{26}$. As Elliott Lieb remarked, a chunk of any such matter "would be very unpleasant stuff to have hanging around the house." 42 .

Some mechanism must exist to offset the quadratic dependence of the Coulomb energy on $N+M$. The first conclusive proof of stability of the second kind was due to Dyson and Lenard in the late $1960 \mathrm{~s}^{43}$, and it relied critically on a fact that electrons are fermions: the many-electron wavefunction (8) must be antisymmetric under the interchange of $\left(\mathbf{x}_{i}, \sigma_{i}\right)$ and $\left(\mathbf{x}_{j}, \sigma_{j}\right)$ for any $i \neq j$. Dyson was also able to show that bosonic matter is not stable ${ }^{44}$ : The ground state energy $E_{0}$ of $2 N$ charged bosons, $N$ with

\footnotetext{
${ }^{41}$ See Loss (2005) p. 53.

${ }^{42}$ Lieb (1990), p. 23.

${ }^{43}$ Dyson and Lenard $(1967,1968)$.

${ }^{44}$ Dyson (1967). For further details see Loss (2005), p. 7.
} 
charge +1 and $N$ with charge -1 satisfies

$$
E_{0} \leq-C N^{7 / 5} \text {. }
$$

For such matter, its volume would decrease with $N$; more particles would take up less space. Again, in Elliott Liebs' words:

... the imposition of the Pauli exclusion principle raises $\left[E_{0}\right]$. The miracle is that it raises $\left[E_{0}\right]$ enough so that the stability of the second kind holds. While it is easy to say that $\psi$ must be antisymmetric ... it is not easy to quantify the effect of antisymmetry. Even the experts have difficulty, for it is not easy to think of an antisymmetric function of a large number of variables. ${ }^{45}$

An alternative, and relatively simple proof of stability of the second kind for fermionic matter was provided by Lieb and Thirring in 1975. This proof exploited features of the Thomas-Fermi theory of the electronic structure of many-body systems ${ }^{46}$, which puts emphasis on the single particle density function $\rho_{\Psi}$ rather than the wavefunction:

$$
\rho_{\Psi}\left(\mathbf{x}_{1}\right)=\sum_{i=1}^{N} \sum_{\sigma_{1}, \ldots, \sigma_{N}} \int_{\mathbb{R}^{3 N-1}}\left|\Psi\left(\mathbf{x}_{1}, \sigma_{1} ; \ldots ; \mathbf{x}_{N}, \sigma_{N}\right)\right|^{2} \mathrm{~d} \mathbf{x}_{2} \ldots \mathrm{d} \mathbf{x}_{N} .
$$

Lieb and Thirring showed first that there is a many-body analogue of (6) for wavefunctions of unit norm (so $\left.\int_{\mathbb{R}^{3}} \rho_{\psi}(\mathbf{x}) \mathrm{d} \mathbf{x}=N\right)$ :

$$
\langle T\rangle_{\psi} \geq \frac{\hbar^{2}}{2 m} \frac{K}{q^{2 / 3}} \int_{\mathbb{R}^{3}} \rho_{\Psi}(\mathbf{x})^{\frac{5}{3}} \mathrm{~d} \mathbf{x},
$$

where

$$
\langle T\rangle_{\psi}=\frac{\hbar^{2}}{2 m} \sum_{\sigma_{1}, \ldots, \sigma_{N}} \sum_{i=1}^{N} \int_{\mathbb{R}^{3 N}}\left|\nabla_{\mathbf{x}_{i}} \Psi\left(\mathbf{x}_{1}, \sigma_{1} ; \ldots ; \mathbf{x}_{N}, \sigma_{N}\right)\right|^{2} \mathrm{~d} \mathbf{x}_{1} \ldots \mathrm{d} \mathbf{x}_{N} .
$$

It is speculated that the best constant in the Lieb-Thirring inequality (11) is $K=(3 / 5)\left(6 \pi^{2}\right)^{2 / 3}$. Note that if the wave function is such that the single particle density is distributed in $N$ equal disjoint bumps across space then the right side of (11) is proportional to $N$.

More generally, Lieb and Thirring went on to prove stability of the second kind, $E_{0} \geq-C(N+M)$, with a much improved value of the constant $C$ in relation to that of Dyson and Lenard. ${ }^{47}$ (In fact, this inequality has

\footnotetext{
${ }^{45}$ Lieb (1990), p. 15.

${ }^{46}$ One such feature is the important result originally due Teller that atoms do not bind: the energy of a system of electrons and nuclei is minimised if the atoms are infinitely far apart and neutral.

${ }^{47}$ Lieb and Thirring (1975).
} 
been shown to hold with $N+M$ replaced by $M{ }^{48}$ ) A further comforting consequence of this result is that fermionic matter in its ground state is indeed bulky: its volume is proportional to $N{ }^{49}$

So far we have been discussing the problem of avoiding implosion of bulk matter associated with the near-range singularity in the Coulomb potential. But in the treatment of macroscopic systems which purport to have typical thermodynamic behaviour, it is also necessary to account for the non-trivial fact that such systems don't explode! Here we are concerned with the longrange behaviour of the Coulomb potential, and the demonstration that $E_{0} / N$ has a limit as $N \rightarrow \infty$. Happily, a proof of the existence of a thermodynamic limit in this sense was provided by Lieb and Lebowitz in $1972 .{ }^{50}$ It is another interesting chapter in the story of the stability of matter, but once stability of the second kind is established, it turns out that little further quantum mechanics is needed to complete it. ${ }^{51}$

Let's go back to 1931, when Ehrenfest raised the question as to why an atom of lead, for example, doesn't pack more of its 82 electrons into the orbits close to the nucleus, and so be smaller than it appears to be. He realized the size of the atom, and the bulky nature of matter generally, must have something to do with the Pauli exclusion principle. He addressed the following point to its originator:

You must admit, Pauli, that if you would only partially repeal your prohibitions, you could relieve many of our practical worries, for example the traffic problem on our streets. ${ }^{52}$

Thanks to the hard work of later quantum physicists, we know why Ehrenfest was right. Matter is both stable and bulky because the many-electron wavefunction has a key property when the electrons are not confined to two dimensions: it is antisymmetric under exchange of particle indices.

\section{Remarks on QBism}

(i) QBism is nothing if not ambitious. It "corrects a profound misconception in our general view of science, which led us into major confusion in the 20th century." 53 This misconception is that science is about an external reality

\footnotetext{
${ }^{48}$ See Lieb and Sieringer (2010).

${ }^{49}$ Lieb and Thirring (1976).

${ }^{50}$ Lieb and Lebowitz (1972).

${ }^{51}$ See Lieb (1976), section V.

${ }^{52}$ Quoted in Dyson (1976); see also Lieb (1990), p. 25.

${ }^{53}$ Fuchs et al. (2014).
} 
that can and should be described without introducing the human agent the "subject". QBism regards the root cause of this misconception to be the failure to fully appreciate that, in the words of David Mermin, a convert to QBism, "scientific pictures of the world rest on the private experiences of individual scientists", and "each of us has a view of our world that rests entirely on our private personal experience." 54 QBism puts the "subject" alongside the "object" (the world) in scientific discourse:

According to QBism, quantum mechanics is a tool anyone can use to evaluate, on the basis of ones past experience, one's probabilistic expectations for one's subsequent experience. ... [Q] mechanics itself does not deal directly with the objective world; it deals with the experiences of that objective world that belong to whatever particular agent is making use of the quantum theory. ${ }^{55}$

Now I cannot think that the ultimate grounding for this view is the innocuous notion that science is an attempt by humans to make sense of the world given to us through our senses, and that science is a human construct. On the contrary, it seems that the basis of a variant of Berkeleyian idealism which suffuses QBism (an admittedly provocative claim, but see (v) below) may be more directly linked to the subjectivist or "personalist" interpretation of probability that is central to the theory.

Since probabilities are the personal judgments of an agent, it follows that a quantum state assignment is also a personal judgment of the agent assigning that state. ${ }^{56}$

QBism adopts a subjectivist stance on probability in physics, inspired principally by the writings of Bruno de Finetti. For the purposes of this essay, I have no objections to it; indeed I largely share it. ${ }^{57}$ So suppose we accept the premiss that probabilities are, loosely speaking, related to betting quotients that rational agents place on chance events. No agents, no probabilities. What I question is the further inference in QBism that our scientific reasoning should primarily be about our personal experiences, our "beliefs", and not the objective world. E. T. Jaynes was perhaps the most prominent

\footnotetext{
${ }^{54}$ Mermin (2016).

${ }^{55}$ Fuchs et al. (2014).

${ }^{56}$ Fuchs et al. (2014).

${ }^{57} \mathrm{My}$ own views on probability are partly spelt out in Brown (2011). But for a critique of the subjectivist interpretation of probability in the context of QBism, see Timpson (2008).
} 
and astute defender of a Gibbsian approach to classical statistical mechanics based on a subjectivist interpretation of probability. Jaynes was also a fan of de Finetti. It did not lead him to say that statistical mechanics is essentially about his and other agents' personal expectations; he never concluded that that theory "does not deal directly with" the world of molecules in gases, and stars in galaxies, etc., for which it provides dynamics. There is more to statistical mechanics than just the probabilities, and arguably it is no different in quantum theory. ${ }^{58}$

(ii) There are two principles of probabilistic updating in QBism. Besides the Bayesian updating associated with the registration of measurement outcomes, the wavefunction is also updated between measurements: it evolves according to the Schrödinger equation, whether there are external forces or not. Now it is a recurring theme in QBism, to which we return in (v) below, that our beliefs about likely perceived events in the future are a result of our interacting with the world.

In QBism the outcome of a measurement is the experience the world induces back in the user who acts on the world. ${ }^{59}$

Suppose then that the quantum system in question evolves freely over a finite interval of time, in which there are no measurements of the system taking place, and so no "experimental kicks". The notion that an agent's subjective quantum probabilities related to the system undergo a non-trivial change in this interval - determined by a specific Hamiltonian that carries no information about previous or future measurements - seems mysterious to me. The agent might even be asleep! Quantum process tomography, involving intitial and final measurements, confirms that time evolution in such cases exists, but does not account for its happening. According to QBism there is no ontic state objectively evolving and dragging the probabilities along with it, in analogy with the Liouville evolution of the probability distribution in classical statistical mechanics. ${ }^{60}$ It is as if von Neumann's two

\footnotetext{
${ }^{58}$ For a clear account of why Jaynes thought equilibrium statistical mechanics works, which has little to do with the choice of probability assignments, see Jaynes (1957). Fuchs (2016) himself states that "there is more to quantum mechanics than just three isolated terms (states, evolution, and measurement)", but he has something quite different in mind; see (vi) below.

${ }^{59}$ Mermin (2016).

${ }^{60}$ Consider the claim made recently by Leifer (op. cit., p. 71 ) that in the epistemic view of the state in quantum mechanics "the appropriate analogies are between quantum states and probability distributions, and between the Schrödinger equation and Liouville's equation." This holds for the Einstein version of the epistemic state, but not for QBism.
} 
motions in quantum mechanics have reappeared in a different guise! The difference now is that the mystery lies with the unitary evolution.

(iii) QBists make a point of distinguishing between information and belief; they argue that it is the progression of the latter that quantum theory describes. I suppose a typical agent's past experience will, if the agent is sufficiently clued up, believe that the quantum state will evolve according to the Schrödinger equation between measurements. But this possible response to the problem posed in (ii) is unconvincing - it cuts no ice in relation to the key question as to whether the wavefunction itself is "belief".

Mermin accepts that

My reification of the concepts I invent, to make my immediate sense of $[$ sic $]$ data more intelligible, is a useful tool of day-to-day living. But when subtle conceptual issues are at stake, related to certain notoriously murky scientific concepts like quantum states, then we can no longer refuse to acknowledge that our scientific pictures of the world rest on the private experiences of individual scientists. ${ }^{61}$

The arguments given in section 2 above are attempts to show, following Timpson's 2008 lead, that this unevenness is unwarranted, even for a proponent of Bayesianism. Wavefunctions and their properties allow us to make sense of our experiences not only in the laboratory but in certain day-to-day phenomena, just as the concept of other agents does. As Elliott Lieb wrote in 1990:

But we also see the effects of quantum mechanics, without realizing it, in such mundane facts about stability as that a stone is solid and has a volume which is proportional to its mass, and that bringing two stones together produces nothing more exciting than a bigger stone. ${ }^{62}$

(iv) Mermin writes:

Some claim, for example, that quantum states were evolving (and even collapsing) in the early universe, long before anybody

Timpson (2008), section 2.2, is also concerned with the issue of objective evolution of the state in QBism, but to different ends.

${ }^{61}$ Mermin (2016).

${ }^{62}$ Lieb (1990), p. 1. See also the two first epigraphs at the start of the present paper. 
existed to assign such states. But the models of the early universe to which we assign quantum states are models that we construct to account for contemporary astrophysical data. ${ }^{63}$

Yet it is hard to avoid the queston: what was evolving in the early universe, if not quantum states? It is not that the question is ill-posed in QBism; it is rather that it leads nowhere. Fuchs tells us that the universe is "made of something else than quantum states", ${ }^{64}$ but details are not thick on the ground.

An analogous scenario suggests itself in the spatial, rather than temporal domain. Using the Pauli exclusion principle, Chandrasekhar famously explained in 1931 the gravitational stability and instability of stars in their late evolutionary phase as white dwarfs. ${ }^{65}$ I take it that the QBist is committed to saying that such stellar models are only constructed to account for what humans see in their telescopes; so the quantum states of stars are no more than the figments of the highly trained imaginations of astrophysicists. Again, we seem to be left with an explanatory gap.

It is worth noting at this point the reason QBists consider Bell's criticism of the Copenhagen interpretation - recall section 2 above - not to apply to their theory. It is that what QBism encompasses are not just the agent's experiences of the "piddling" results of measurements in Earth-bound scientific laboratories. The theory allows

each of us to take the scope of physics to be any of the manifold aspects of our own experience ... ${ }^{66}$

And

Users are making measurements more or less all the time more or less everywhere. Every action on her world by every user constitutes a measurement, and her experience of the world's reaction is its outcome. ${ }^{67}$

I cannot help but find this response to Bell unconvincing. Generalising the notion of measurement to include the myriad experiences of agents gained outside the laboratory will in large part lead to theories, or "personal modes

\footnotetext{
${ }^{63}$ Mermin (2016).

${ }^{64}$ Fuchs (2016), footnote 5 .

${ }^{65}$ Chandrasekhar (1931).

${ }^{66}$ Fuchs et al. (2014).

${ }^{67}$ Mermin (2006).
} 
of thought" that have very little to do with quantum physics. More importantly, I suspect Bell - the inventor of the word beable - would have thought that to circumscribe physics to what is going on in the minds of human beings, even when outside laboratories, would still be "to betray the great enterprise". I suspect he wanted science to try to tell us, amongst many other things, what actually happened in the early universe, and what has actually gone on inside stars since then.

(v) Earlier, I used the description "a variant of Berkeleyian idealism" in relation to QBism. This may well seem inappropriate. Bishop Berkeley did not believe in a reality external to human perceptions, apart from God. QBists do. Indeed, Fuchs says that QBism and related views "should be regarded as attempts to make a deep statement about the nature of reality." 68 So it would seem that QBism is not strictly idealism in Berkeley's sense; it does not "deny the existence of an observer-independent reality", as Leifer claimed (see section 3.3 above). But I find the ineffable nature of the external world in QBism troubling, and it is this concern that leads me to make the analogy with George Berkeley's metaphysics.

A key notion in the theory is, as was mentioned in (i) above, that of the interaction between the agent and the world. Here is the way Fuchs and Schack put the point:

$[\mathrm{O}]$ ne...might say of quantum theory, that in those cases where it is not just Bayesian probability theory full stop, it is a theory of stimulation and response .... The agent, through the process of quantum measurement stimulates the world external to himself. The world, in return, stimulates a response in the agent that is quantified by a change in his beliefs - i.e., by a change from a prior to a posterior quantum state. Somewhere in the structure of those belief changes lies quantum theory's most direct statement about what we believe of the world as it is without agents. ${ }^{69}$

In Mermin's words:

Science is about the interface between the experience of any particular person and the subset of the world that is external to that particular user. ${ }^{70}$

\footnotetext{
${ }^{68}$ Fuchs (2016), p. 1.

${ }^{69}$ Fuchs and Schack (2004).

${ }^{70}$ Mermin (2016).
} 
Let us remind ourselves why the QBist needs to postulate such an interface, since after all we cannot be certain such a world external to our subjective experiences exists. Here is Fuchs' reason:

I would say all our evidence for the reality of the world comes from without us, i.e., not from within us. We do not hold evidence for an independent world by holding some kind of transcendental knowledge. ... We believe in a world external to ourselves precisely because we find ourselves getting unpredictable kicks (from the world) all the time. ${ }^{71}$

Not quite. What we find ourselves getting is forever changing subjective experiences. Berkeley, and many other thinkers over the ages, have not been content to leave it at that. They have looked for an explanation of these more-or-less structured experiences (predictable or otherwise), and in particular, an explanation of the correlations between the experiences of different agents. Berkeley chose the intervention of God; QBists (and scientists generally) choose that of the world. I am willing to grant they are not the same thing, but in the case of QBism there are, I think, analogies that are striking. I will try to spell this out.

Berkeley's famous dictum esse est percipi (aut percipere) - to be is to be perceived (or to perceive) - was based on the claim that the action of matter on mind is inexplicable, implying that to postulate the existence of matter is pointless:

... though we give the materialists their external bodies, they by their own confession are never the nearer knowing how our ideas are produced: since they own themselves unable to comprehend in what manner body can act upon spirit, or how it is possible it should imprint any idea in the mind. Hence it is evident the production of ideas or sensations in our minds, can be no reason why we should suppose matter or corporeal substances, since that is acknowledged to remain equally inexplicable with, or without this supposition. ${ }^{72}$

Modern philosophers of mind tend not to follow Berkeley in rejecting the material world, but they are certainly divided on how to make sense of the relationship between conscious states and the underlying neurophysiological states of the brain of the agent in question. The problem of how "qualia" -

\footnotetext{
${ }^{71}$ Fuchs 2002b), also quoted in Fuchs (2016).

${ }^{72}$ Berkeley (1710).
} 
the introspectively accessible, phenomenal aspects of our mental experiences - relate to the physical world both in the brain and in its environment is a central contentious issue in the philosophy of mind. ${ }^{73}$ But it is widely accepted that some important kind of connection exists between mental experiences and physical brain states, and that physics has something to say at least in principle about how the latter are affected by the external environment. Is there space in QBism for something like this picture?

I take it that the things "external" to the agent (call her Alice), such as atoms, tables and chairs and other agents (such as Bob), are part of what QBists call the world. Consider then the following way QBists distinguish their position from that of Bohr:

Acting as an agent, Alice can use the formalism of quantum mechanics to model any physical system external to herself. QBism directs her to treat all such external systems on the same footing, whether they be atoms, enormous molecules, macroscopic crystals, beam splitters, Stern-Gerlach magnets, or even agents other than Alice. ...

... But because Alice can treat Bob as an external physical system, according to QBism she can assign him a quantum state that encodes her probabilities for the possible answers to any question she puts to him. ${ }^{74}$

Quantum mechanics is seen then to "model" physical systems like atoms and agents, but the notion is a subtle one. To repeat, "... quantum mechanics itself does not deal directly with the objective world; it deals with the experiences of that objective world ...". The modelling is done purely by way of specifying quantum states and their dynamical behaviour, the states themselves being "beliefs" belonging to a single agent. The external physical systems float free of the quantum formalism. No describable objective attributes can be assigned to these systems in QBism, because, as we have seen, the universe is made of something other than quantum states, and quantum states are all we have in the formalism of quantum mechanics.

So how are we to understand the nature of the interface between agents and the world that plays such an important role in QBism and specifically its claims to be "realist"? Think first of Alice concerning herself with Bob's interaction with things in the world around him. Insofar as she is equipped with knowledge of quantum mechanics, and provides a formal model for this

\footnotetext{
${ }^{73}$ For an introduction to the problem of qualia, see Tye (2016).

${ }^{74}$ Fuchs et al. (2014).
} 
interaction, it will not strictly be between agents like Bob and other parts of the external world but between quantum states associated with these systems. These are all part of Alice's "personal mode of thought". What about Alice's own interface with the world? I quote Fuchs, Mermin and Schack:

In QBism the only phenomenon accessible to Alice which she does not model with quantum mechanics is her own direct internal awareness of her own private experience. ... Her awareness of her past experience forms the basis for the beliefs on which her state assignments rest. And her probability assignments express her expectations for her future experience. ${ }^{75}$

This seems to go beyond acknowledgment of the mystery of qualia (if mystery is the right word); there seems to be an indication that there is no model based on the quantum formalism that Alice can construct to account even for the states of the physical brain substrate underlying her own personal experiences. Indeed, it is not clear to me how such a thing could exist in QBism, in which assignment of quantum states rests on beliefs, which in turn rest on subjective experience. But even if Alice can somehow assign a quantum state to her own brain, the nature of its interaction with the external world is opaque because, again, for the QBist the world is itself not made of wavefunctions. What does it mean then to say that we find ourselves getting kicks from the world? Here is a variation on the QBism theme:

When an experimentalist reaches out and touches a quantum system - the process usually called quantum 'measurement' that process gives rise to a birth. It gives rise to a little act of creation. And it is how those births or acts of creation impact the agent's expectations for other such births that is the subject matter of quantum theory. ${ }^{76}$

The language is colourful, and the recent term "participatory realism" Fuchs has used to describe QBism ${ }^{77}$ is alluring, but the nature of the agent-world interface in QBism seems to be entirely obscure from a physics perspective for that agent. How, in particular, does Alice know that she interacts only

\footnotetext{
${ }^{75}$ Fuchs et al. (2014).

${ }^{76}$ Taken from the introduction to a 2004 lecture by Christopher Fuchs, and reproduced in Fuchs (2016).

${ }^{77}$ Fuchs (2016).
} 
with a "subset" of the world? What does subset in this context even mean? Why does the world react to and act on Bob in a way similar to its interaction with Alice? If Alice applies physical notions like locality (no action-at-adistance), and divisibility into subsystems, to the external world that is purportedly acting on her, she does so with no clear justification. That part of QBism which relates to "a theory of stimulation and response" between the agent and the world is not grounded in known physics.

For Berkeley, the nature of God's action in creating living minds is a mysterious affair. That's the way it is with God. I fail to see how the action of the external world on human agents in QBism is any less mysterious.

(vi) It would arguably be a step in the right direction if QBists, in their zeal for realism, were to conclude that understanding the universe is the true aim of physics, and that current quantum theory, as they see it, is a stop-gap. If I have understood him correctly, Fuchs has gone some way to adopting this stance.

Ultimately, as physicists, it is the quantum world for which we would like to say as much as we can, but that is not our starting point. Quantum theory rests at a level higher than that. To put it starkly, quantum theory is just the start of our adventure. The quantum world is still ahead of us.

But recall the self-proclaimed revolutionary nature of QBism and its philosophy of nature:

We bring QBism to the readers attention because it corrects a profound misconception in our general view of science, which led us into major confusion in the 20th century. Now that we are well into the 21st and we all agree that quantum mechanics works spectacularly well for every practical purpose, surely it is time to expand our ancient view of the nature of science, to dispel the murkiness that has obscured the foundations of the theory for too long. ${ }^{78}$

Whether this is is consistent with the view that quantum theory is merely a stop-gap is surely debatable, as is the question as to how revolutionary QBIsm really is. Given the nature of modern philosophy of science at least in the Anglophone tradition, it is easy to overlook the existence of a major idealist trend in natural philosophy that might be said to have started with

\footnotetext{
${ }^{78}$ Fuchs et al. (2014).
} 
Leibniz, Berkeley and Kant, and which gives, in varying ways, the "subject" a prominent role in the understanding of scientific thinking.

What I find startling is Fuchs' recent comparison of QBism with Einstein's philosophy of science, in which he "cannot see any way in which the program of QBism has ever contradicted what Einstein calls the program of "the real". ..."79 This remarkable claim is worth examining.

If the QBist is truly to treat atoms and laboratory equipment and human agents on the same footing, then elements of the familiar macroworld, as much as elements of the microworld, are to be treated as "concepts" in "a personal mode of thought", i.e. theory, "that any agent can use to organize her own experience". As we have seen, the QBist is resolutely silent on the precise nature of the external world. Better to say it is as if the world is populated by such entities as atoms, tables and chairs, according to our best theories. As I understand Einstein's brand of scientific realism, it is indeed not far from this "as-if" reconstruction of QBism. Einstein's view was that in explaining the structure of human experience (what he called the "subjective factor"), the scientist is charged with coming up with coherent models of an external reality involving mind-independent elements (the "objective factor"), including presumably the constituents of the brains of sentient beings. For Einstein, who had a life-long interest in philosophy, this realist commitment is tentative. As he said,

... the "real" in physics is to be taken as a type of program, to which we are, however, not forced to cling a priori. ... ${ }^{80}$

Einstein was aware that the program could fail in principle; it was a dogma about which he was not dogmatic, though he recognised of course that so far the historical record has been encouraging. But were he asked if the fundamental objects in a successful model of some domain in physics actually correspond to the relevant part of the actual world, Einstein's answer would be a smile. He too would not be drawn into a discussion of what transcends the "as if" world. ${ }^{81}$ In philosophical jargon, Einstein was an advocate of a deflationary theory of truth, not a correspondence theory.

But I think there is an essential difference between Einstein's position and "as if" QBism, and it has to do with the scope of the "objective factor":

\footnotetext{
${ }^{79}$ Fuchs (2016).

${ }^{80}$ Einstein (1970), p. 674 . This is part of a longer Einstein quotation found in Fuchs (2016).

${ }^{81}$ See Einstein (1970), p.680, where Einstein attributes this position to the influence of Immanuel Kant. For further references to Einstein's realist philosophy, and to that of commentators, see Brown and Lehmkuhl (2016), footnote 4.
} 
The ... objective factor is the totality of such concepts and conceptual relations as are thought of as independent of experience, viz., of perceptions. So long as we move within the thus programmatically fixed sphere of thought we are thinking physically. ${ }^{82}$

I find it hard to reconcile this reasoning with the notion that quantum mechanics is a complete theory and, according to Fuchs,

... the best understanding of quantum theory is obtained by recognizing that quantum states, quantum time-evolution maps, and the outcomes of quantum measurements all live within what Einstein calls the subjective factor. ${ }^{83}$

Of course, for the post-1927 Einstein the wavefunction is, as we have seen, essentially a probability distribution over hidden ontic states; it is (at least) these ontic states that correspond to a "concept" that is "independent of experience", if we are "thinking physically". According to Einstein, orthodox quantum mechanics is incomplete precisely because it does not specify what such ontic states are. The idea that quantum physics can do without them altogether seems to me to be antithetical to Einstein's program, metaphysically shy though it is.

Fuchs strongly resists criticisms to the effect that QBism is non-realist. In a 2016 paper, he addresses the authors of such criticisms and accuses them of indulging in a non sequitur:

This is because, if any of these cads were to take a moment to think about it, they would recognize that there is more to quantum mechanics than just three isolated terms (states, evolution, and measurement) - there's the full-blown theory that glues these notions together in a very particular way, and in a way that would have never been discovered without empirical science.

I am not sure I entirely understand what is meant here, but presumably the glue in QBism has something to do with the role of the external world in underpinning the notion of experience itself. But this world is not the "as if" world populated by well-defined mind-independent concepts in physical theory, as Einstein understood it. The ineffable world of QBism would, I submit, have held little interest for Einstein.

\footnotetext{
${ }^{82}$ Einstein (1970), pp. 673-4; again this is part of the longer quotation given in Fuchs (2016).

${ }^{83}$ Fuchs (2016).
} 


\section{Acknowledgments}

I thank the organisers of the XII International Ontology Congress for the kind invitation to contribute to these proceedings. I am also grateful to David Wallace for useful remarks, and to Rhys Borchert and particularly Christopher Timpson for invaluable critical comments on the first draft of this paper. None should be taken to endorse the arguments presented here.

\section{References}

J.S. Bell (1990), "Against 'measurement'", Physics World 3(8), 33-40.

M. Bell and S. Gao (eds.) (2016) Quantum Nonlocality and Reality, Cambridge University Press, Cambridge UK.

G. Berkeley (1710), "Of the Principles of Human Knowledge: Part 1", republished in The Works of George Berkeley, Bishop of Cloyne. A.A. Luce and T.E. Jessop (eds.), London: Thomas Nelson and Sons.

H.R. Brown (2011), "Curious and sublime: the connection between uncertainty and probability in physics", Philosophical Transactions of the Royal Society A 369, 1-15. http://philsci-archive.pitt.edu/8571/

H.R. Brown., C. Dewdney and G. Horton, "Bohm particles and their detection in the light of neutron interferometry", Foundations of Physics 25, 329-34.

H.R. Brown and D. Lehmkuhl (2016), "Einstein, the reality of space, and the action-reaction principle", in Einstein, Tagore and the Nature of Reality, Partha Ghose (ed.), Routledge, London and New York, 2016; pp. 9-36. arXiv:1306.4902v1.

H.R. Brown and J. Read, (2016), "Clarifying possible misconceptions in the foundations of general relativity", American Journal of Physics, 84(5), 327-334.

H.R. Brown, J. Summhammer, R. Callaghan and P. Kaloyerou (1992), "Neutron interferometry with antiphase modulation", Physics Letters A 163, 21-25.

H.R. Brown and D. Wallace (2005), "Solving the measurement problem: de Broglie-Bohm loses out to Everett", Foundations of Physics 35, 517-540. quant-ph/0403094; PITT-PHIL-SCI 1659.

J.W.M. Bush (2015), "The new wave of pilot-wave theory", Physics Today 68(8), 47-53; doi: 10.1063/PT.3.2882.

Callender, C. (2017), "The Redundancy Argument Against Bohm's Theory" manuscript available at http://philosophyfaculty.ucsd.edu/faculty/ccallender/publications.shtml. 
S. Chandrasekhar (1931), "The density of white dwarfstars", Philosophical Magazine 11, 592-596.

F.J. Dyson (1967), "Ground-state energy of a finite system of charged particles", Journal of Mathematical Physics 8, 1538-1545.

F.J. Dyson and A. Lenard (1967), "Stability of matter. I.", Journal of Mathematical Physics 8, 423-434.

F.J. Dyson and A. Lenard (1968), "Stability of matter. I.,", Journal of Mathematical Physics 9, 698-711.

D. Dürr, S. Goldstein, and N. Zanghi (1997), "Bohmian Mechanics and the Meaning of the Wave Function", in R. S. Cohen, M. Horne, and J. Stachel (eds.), Experimental Metaphysics: Quantum Mechanical Studies for Abner Shimony, vol. 1, Boston Studies in the Philosophy of Science 193; Dordrecht: Kluwer, pp. 25-38.

A. Einstein (1970), "Remarks Concerning the Essays Brought Together in This Co-operative Volume", in Albert Einstein: Philosopher-Scientist, Volume Two, edited by P. A. Schilpp, Open Court, La Salle, Illinois; third edition.

R.L. Frank (2011), "Sobolev inequalities and uncertainty principles in mathematical physics: part 1", Lecture Notes (LMU Munich) (available at https://web.math.princeton.edu/?rlfrank/sobweb1.pdf)

J. Frohlich (2009), "Spin, or actually: Spin and Quantum Statistics", in The Spin, B. Duplantier, J.-M. Raimond and V. Rivasseau (eds.); Birkhäuser Verlag Basel/Switzerland; pp. 1-60.

C.A. Fuchs (2016), "On Participatory Realism", arXiv:1601.04360v3 [quant$\mathrm{ph}]$.

C.A. Fuchs (2002a), "Quantum Mechanics as Quantum Information (and only a little more)", arXiv:quant-ph/0205039v1.

C.A. Fuchs (2002b), "The Anti-Vaxjo Interpretation of Quantum Mechanics", http://arxiv.org/abs/quant-ph/0204146:

C.A. Fuchs, N.D. Mermin, and R. Schack (2014), "An Introduction to QBism with an Application to the Locality of Quantum Mechanics", American Journal of Physics 82(8), 749-754.

C.A. Fuchs and R. Schack (2013), "Quantum-Bayesian coherence", Reviews of Modern Physics 85, 1693-1714.

S. Gao (2017), Meaning of the wavefunction. In search of the ontology of quantum mechanics, Cambridge University Press, Cambridge UK; arXiv:1611.02738v1 [quant-ph].

G.H. Hardy (1920), "Note on a theorem of Hilbert". Mathematische Zeitschrift 6 (3-4): 314317. 
N. Harrigan and R.W. Spekkens (2010), "Einstein, incompleteness, and the epistemic view of quantum states", Foundations of Physics 40(2):125-157. arXiv:0706.2661, doi:10.1007/s10701- 009-9347-0.

R. Healey (2016), "Quantum-Bayesian and Pragmatist Views of Quantum Theory", https://plato.stanford.edu/entries/quantum-bayesian/

E.T. Jaynes (1957), "Information Theory and Statistical Mechanics", Physical Review 106(4), 620-630.

P. Kaloyerou and H.R. Brown (1992), "On neutron interferometer partial absorption experiments", Physica B 176, 78-92.

M.S. Leifer (2014), "Is the Quantum State Real? An Extended Review of $\psi$-ontology Theorems", Quanta 3, 67-155.

E.H. Lieb (1976), "The Stability of Matter", Reviews of Modern Physics 48, 553-569.

E.H. Lieb (1990), "The stability of matter: from atoms to stars", Bulletin of the American Mathematical Society (N.S.) 22 (1), 1-49.

E.H. Lieb and J.L. Lebowitz (1972), "The constitution of matter: Existence of thermodynamics for systems composed of electrons and nuclei", Advances in Mathematics 9, 316-398.

E.H. Lieb and R. Seiringer (2010), Stability of Matter in Quantum Mechanics, Cambridge University Press, Cambridge.

E.H. Lieb and W. Thirring (1975), "Bound for the Kinetic Energy of Fermions Which Proves the Stability of Matter", Physical Review Letters 35 (11), 687-689.

E.H. Lieb and W. Thirring (1976), "Inequalities for the moments of the eigenvalues of the Schrödinger Hamiltonian and their relation to Sobolev inequalities", in E. Lieb, B. Simon, A. Wightman (eds.), Studies in Mathematical Physics, Princeton University Press, pp. 269-303.

J.M. Leinaas and J. Myrheim (1977), "On the theory of identical particles", Il Nuovo Cimento B 37(1), 1-23.

M. Loss (2005), Stability of Matter, http://www.math.lmu.de/ lerdos/WS08/QM/lossstabmath.pdf.

T. Maudlin (2010), "Can the World be Only Wavefunction?", in Many Worlds? Everett, Quantum Theory, 8 Reality, S. Saunders, J. Barrett, A. Kent, and D. Wallace (eds.), Oxford University Press, Oxford; 121-143.

D. Mermin (2016), "Why QBism is not the Copenhagen interpretation and what John Bell might have thought of it", Quantum [Un]Speakables II Part of the series The Frontiers Collection, Springer-Verlag, pp 83-93. arXiv:1409.2454V1 [quant-ph]

E. Merzbacher (1962), "Single Valuedness of Wave Functions", American Journal of Physics 30(4), 237-247. 
W.C. Myrvold (2015), "What is a wavefunction?", Synthese 192(10), 32473274.

A. Ney (2015), "Fundamental Physical Ontologies and the Constraint of Empirical Coherence: A Defense of Wave Function Realism", Synthese 192(10), 3105-3124.

R.E. Prange and S.M. Girvin (eds.) (1990), The Quantum Hall Effect, Graduate Texts in Contemporary Physics, Springer-Verlag.

M.F. Pusey, J. Barrett and T. Rudolph (2012), "On the reality of the quantum state", Nature Physics 8, 475-478. arXiv:1111.3328, doi:10.1038/nphys2309.

H Putnam (1979) "Is Logic Empirical?". The Logico-Algebraic Approach to Quantum Mechanics, 181-206.

R. Seiringer (1990), "Inequalities for Schrödinger Operators and Applications to the Stability of Matter Problem", http://www.ueltschi.org/AZschool/notes/RobertSeiringer.p

E. Squires, L. Hardy and H.R. Brown (1994), "Non-locality from an analogue of the quantum Zeno effect", Studies in History and Philosophy of Science 25(3), 425-435.

S.L. Sobolev (1938), "On a theorem of functional analysis" (in Russian). Mat. Sb. 46, 471-497. English transl.: American Mathematical Society, Transl., II. Ser. 34, 39-68,1963.

P. Szriftgiser, D. Gury-Odelin, M. Arndt, and J. Dalibard (1996), "Atomic Wave Diffraction and Interference Using Temporal Slits", Physical Review Letters 77(1), 4-7.

W. Thirring (1986), "Stability of matter", in V. Gorini and A. Frigerio (eds.), Fundamental Aspects of Quantum Theory, Plenum Press, New York; pp. 343-354.

M. Tye (2016), "Qualia", The Stanford Encyclopedia of Philosophy (Winter 2016 Edition), Edward N. Zalta (ed.), URL = ¡https://plato.stanford.edu/archives/win2016/entries/q

C. Timpson (2008), "Quantum Bayesianism: A Study", arXiv:0804.2047v1 [quant-ph].

D. Wallace and C. Timpson (2010), "Quantum Mechanics on Spacetime I: Spacetime State Realism", British Journal for the Philosophy of Science 61, 697-727. 